\title{
On Trade-off Between 5G Positioning and mmWave Communication in a Multi-user Scenario
}

\author{
Dileep Kumar, Jani Saloranta, Giuseppe Destino, Antti Tölli \\ Centre for Wireless Communications, University of Oulu, Finland \\ Email: \{dileep.kumar, jani.saloranta, giuseppe.destino, antti.tolli\}@oulu.fi
}

\begin{abstract}
Millimeter-wave communication is considered one of the key enablers for $5 \mathrm{G}$ systems as it contributes to achieving high data rate with very wideband transmission, high beamforming gain and massive MIMO techniques. Further, millimeter-wave technology can also be used for the accurate positioning. However, it is yet unclear how communication and positioning systems can share resources to flexibly fulfill data-rate and quality-of-position requirements, especially, in multiuser scenarios. In this regard, the objective of this paper is to investigate and quantify the trade-off between positioning quality and achievable sum-rate as a function of number of receive antennas and transmitter locations in an uplink multi-user scenario.
\end{abstract}

\section{INTRODUCTION}

Exploding growth in the mobile broadband usage has created a variety of new applications, resulting in an exponential increase in amount of data exchange. To tackle this surge in data traffic and to improve system capacity; significant research efforts have been concentrated towards development of $5 \mathrm{G}$ communication system [1]. One of the key enablers for $5 \mathrm{G}$ is the use of millimiter-Wave (mmWave) spectrum (30-300 GHz), which provides larger system bandwidth and the possibility of packing a large number of antennas in a small form factor for highly directional communication [2]. Thus, 5G systems will provide seamless support for large number of users with diverse Quality-of-Service (QoS), delay and throughput requirements [3]. On the other hand, it have the potential for accurate positioning [4], [5]. In comparison to conventional positioning techniques based on ToA, TDoA and/or RSS, mmWave based directional communication enables determination of user's position from a single access point by processing the training signals in different directional beams [5].

The early work [6] considered downlink power optimization under per-user rate and positioning accuracy constraints, while [7] utilized the user positioning information for efficient beamalignments. Authors in [8] investigated the effect of the training period to the achievable positioning quality and rate, while authors in [9], [10] studied the rate-positioning trade-off for different beam training strategies and provided the tradeoff for a single user scenario. In contrast to previous work, our research objective is to quantify the trade-off between sum-rate and positioning accuracy in uplink for a multi-user mmWave communication system.

The reminder of the paper is organized as follow. First in Section II, we illustrate system architecture and explain the performance metrics as well as provide the formulation of

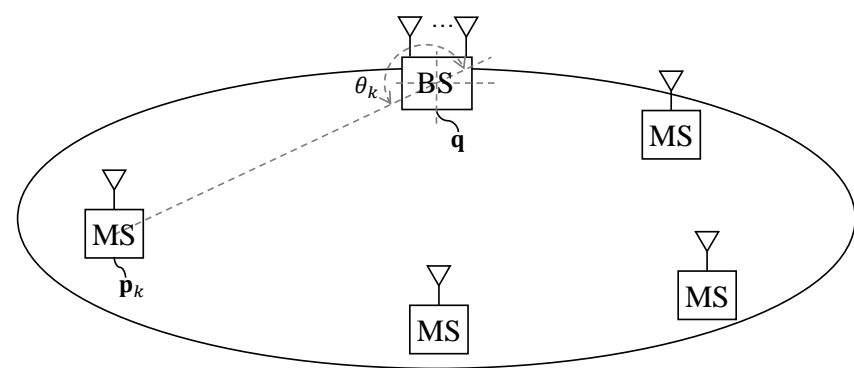

Fig. 1. Uplink system model showing a receiver (BS) at location $\mathbf{q}$ and $k^{\text {th }}$ transmitter (MS) at location $\mathbf{p}_{k}$ with the LOS link and AoA $\theta_{k}$.

the problem. In Section III, we describe the position accuracy using Cramér-Rao Lower Bound. The numerical results are presented in Section IV.

\section{SYSTEM MODEL}

We consider uplink in a mmWave based multi-user singleinput-multiple-output (SIMO) wireless system with a receiver (base station, BS) and $K$ single-antenna transmitters (mobile station, MS). The set of MS is denoted as $\mathcal{K}=\{1,2, \ldots, K\}$. The location of $k^{t h}$ MS is denoted by $\mathbf{p}_{k} \in \mathbb{R}^{2}$ i.e., $\mathbf{p}_{k}=$ $\left[p_{x}^{k}, p_{y}^{k}\right]$. BS is located at the position $\mathbf{q}=\left[q_{x}^{B S}, q_{y}^{B S}\right]$ and equipped with a uniform linear array (ULA) with $N_{B}$ isotropic antenna elements each with gain of $0 \mathrm{dBi}$ and $\lambda / 2$ spacing between any two adjacent elements, where $\lambda$ is the wavelength of carrier frequency. We restrict ourself to the case when BS is equipped with a single baseband, thus one user is served at a given time. We assume $\mathbf{q}$ is known to all nodes in the network, whereas $\mathbf{p}_{k} \forall k \in \mathcal{K}$ is not known.

\section{A. Channel Model}

Due to relatively higher path loss, low-scattering and spatially sparse nature of mmWave channel compared to sub$6 \mathrm{GHz}$ frequency band, channel effectively have 'quasi'-optical propagation properties [2], where line-of-sight (LoS) link is the dominant path. We assume a single-path SIMO channel $\mathbf{h}$ given by

$$
\mathbf{h}=h \mathbf{a}_{R}(\theta),
$$

where $h$ is the complex gain of the dominant path and $\mathbf{a}_{R}(\theta) \in$ $\mathbb{C}^{N_{B}}$ is the receive array response vectors for angle-of-arrival (AoA) $\theta$ with

$$
\left[\mathbf{a}_{R}(\theta)\right]_{m}=e^{j \frac{2 \pi d}{\lambda}(m-1) \sin (\theta)}, m \in\left\{1,2, \ldots, N_{B}\right\} .
$$




\begin{tabular}{|cc}
$\longleftrightarrow T_{t}$ & $T_{f}$ \\
\hline Training Phase & Data Phase \\
\hline
\end{tabular}

Fig. 2. Communication over the frames of duration $T_{f}$, each with a training phase of duration $T_{t}$.

\section{B. Signal Model}

In this sub-section, we develop a generic signal model for the system shown in Figure 1. For simplicity of the analysis, we assume all MSs are allocated orthogonal resources, e.g., by using time-division-multiple-access (TDMA) or frequencydivision-multiple-access (FDMA). Let $f_{k}$ be the fractions of $T_{d}$ resources allocated to $k^{\text {th }} \mathrm{MS}$, such that, $\sum_{i=1}^{K} f_{i} \leq 1$. Let $y_{k}(t)$ be the received signal at the BS from $k^{t h}$ MS, given by

$$
y_{k}(t)=\mathbf{w}^{H} \mathbf{h}_{k} x_{k}\left(t-\tau_{k}\right)+\mathbf{w}^{H} \mathbf{n}(t),
$$

where $\mathbf{w} \in \mathcal{W}$ is a receive beamforming vector obtained by applying appropriate phase-shifts and amplitude scaling to each antenna element, $\tau_{k}=\left\|\mathbf{q}-\mathbf{p}_{k}\right\| / c$ is the propagation delay for $k^{t h}$ MS in the dominant LoS path for speed of light $c, \mathbf{n}(t)$ is additive white Gaussian noise (AWGN) with power spectral density (PSD) $N_{o}, x_{k}(t)$ is the continuous timedomain transmitted signal of bandwidth $B$ and duration $T_{s}$, with $1 / T_{s} \int_{0}^{T_{s}}\left|x_{k}(t)\right|^{2} d t=1, \forall k \in \mathcal{K}$.

Without loss of generality, we will assume that transmitted signal of each MS, $x_{k}(t)$ has flat spectrum, i.e., $X_{k}(\omega)$ is constant with in each transmission interval. Further, each transmission block consist a training phase of duration $T_{t}$ for channel estimation and determining the best transmit-receive beams using orthogonal pilots for each MS. The reminder of duration $T_{d}=T_{f}-T_{t}$ is reserved for the data, as shown in Figure 2.

It should be noted, receive beamforming remain fixed for the complete data reception phase. Therefore, aligning the receive beam only in the direction one MS will results in poor SNR for all other MSs. To avoid this, we first find a compromise receive beam $\tilde{\mathbf{w}}$ that provides comparable SNR by the superposition of best receive beam of each MS and then allocate a portion of $T_{d}$ to each MS based on data-rate requirements.

\section{Performance Metrics}

For each MS, we consider two performance metrics: effective data-rate and positioning accuracy i.e., $R_{k}$ and $Q_{k}$ $\forall k \in \mathcal{K}$. The system design aims at maximizing the minimum data-rate over all MSs for a given position accuracy requirements.

- Effective data-rate $\left(R_{k}\right)$ : Under the considered orthogonal scheme that allocates a fraction of non-overlapping degrees of freedom to each MS, the effective data-rate for $k^{t h}$ MS is give by

$$
R_{k}=B\left(1-\frac{T_{t}}{T_{f}}\right) f_{k} \log _{2}\left(1+\frac{S N R_{k}}{f_{k}}\right),
$$

where

$$
S N R_{k}=\frac{\left|\tilde{\mathbf{w}}^{H} \mathbf{h}_{k}\right|^{2} P}{\sigma^{2}},
$$

$\sigma^{2}=N_{o} B$ is the noise power over signal bandwidth and $P$ is the maximum uplink transmit power for each MS.

- Positioning accuracy $\left(Q_{k}\right)$ : Position accuracy is determined by the Position Error Bound (PEB) and that can be obtained from the Fisher Information Matrix (FIM) [5]. Assuming the training phase is also used for positioning, PEB for the $k^{t h}$ MS is computed as

$$
\begin{aligned}
\mathbf{J}_{k} & =\sum_{\mathbf{w} \in \mathcal{W}} \mathbf{J}_{\tilde{\boldsymbol{\eta}}_{k}}(\mathbf{w}), \\
Q_{k} & =\sqrt{\sigma_{p_{x}^{k}}^{2}+\sigma_{p_{y}^{k}}^{2}},
\end{aligned}
$$

where $\sigma_{p_{x}^{k}}$ and $\sigma_{p_{y}^{k}}$ are obtained from the inverse of FIM [5] as

$$
\begin{aligned}
\sigma_{p_{x}^{k}}^{2} & =\left[\mathbf{J}_{k}^{-1}\right]_{1,1}, \\
\sigma_{p_{y}^{k}}^{2} & =\left[\mathbf{J}_{k}^{-1}\right]_{2,2} .
\end{aligned}
$$

The derivation of $\mathbf{J}_{k}$ will be provided in section III.

\section{Problem Formulation}

The aim of this work is to find an optimal duration for the training phase and allocation of the degrees of freedom in order to satisfy positioning accuracy requirements for each MS, with the objective of common-rate maximization. The problem can be formulated as

$$
\begin{array}{cl}
\underset{T_{t}, f_{i}, R_{o}}{\operatorname{maximize}} & R_{o}\left(T_{t}, f_{i}\right) \\
\text { subject to } & R_{k}\left(T_{t}, f_{k}\right) \geq R_{o}, \\
& Q_{k}\left(T_{t}\right) \leq Q_{t h} \quad \forall k \in \mathcal{K}, \\
& \sum_{i=1}^{K} f_{i} \leq 1, \\
& f_{i} \geq 0 \quad \forall i=1,2, \ldots, K
\end{array}
$$

Note that, constraint (10b) imposes that achievable data-rate for each MS is greater than or equal to the common rate $R_{o}$. This can be attained by optimal sharing the degrees of freedom $T_{d}$ among all active MSs, such that, each MS should get non-overlapping and non-negative fraction of the system resources (10d) and (10e), respectively. Finally, positioning constraint (10c) implies that PEB for each MS is smaller than the threshold positioning accuracy $Q_{t h}$ which is related to the number of receive beams used for the training.

From the above, we can observe the impact of training duration $T_{t}$ on sum-rate and positioning accuracy. For example, use of narrower receive beams at BS will improve positioning accuracy and SNR, but also lead to an increase in training overhead $\left(T_{t}\right)$, thus less time for data reception $\left(T_{f}-T_{t}\right)$ and hence will results in lower sum-rate. Conversely, wider receive beams decrease training overhead, but results in lower positioning accuracy as less Fisher information leads to more PEB. Clearly, there is tension between achievable data-rate 
and the positioning accuracy of each MS. Our goal here is to understand and quantify this trade-off.

\section{PEB COMPUTATION}

In this section, we elaborate on solution of optimization problem (10). The achievable data rate of $k^{\text {th }} \mathrm{MS}$ is given as (4), while PEB in the constraint (10c) is set equal to Cramér-Rao Bound (CRB). The CRB provides a lower bound on variance of an estimator of unknown parameters. In other words, variance of any unbiased estimator is at least as high as the inverse of Fisher information [11]. Our aim here is to find the PEB for all MSs i.e., $Q_{k} \forall k \in \mathcal{K}$. We adopted two step approach: firstly, we derive FIM of the channel parameters such as delay $\boldsymbol{\tau}$, AoA $\boldsymbol{\theta}$, real part of channel coefficients $\mathbf{h}^{R}$ and imaginary part of channel coefficients $\mathbf{h}^{I}$, by scanning of the receive beams during the training phase. Then, using a bijective transformation, we obtain the positioning accuracy.

\section{A. FIM: Channel Parameters}

Let $\boldsymbol{\eta} \in \mathbb{R}^{4 K}$ be vector of unknown channel parameters

$$
\boldsymbol{\eta}=\left[\boldsymbol{\tau}^{T}, \boldsymbol{\theta}^{T}, \mathbf{h}^{R^{T}}, \mathbf{h}^{I^{T}}\right],
$$

consist of vectors of delay, AoA and real and imaginary coefficients of channel, such that, $\boldsymbol{\tau} \doteq\left[\tau_{1}, \tau_{2}, \ldots, \tau_{K}\right]$, $\boldsymbol{\theta} \doteq\left[\theta_{1}, \theta_{2}, \ldots, \theta_{K}\right], \mathbf{h}^{R} \stackrel{\circ}{=}\left[h_{1}^{R}, h_{2}^{R}, \ldots, h_{K}^{R}\right]$ and $\mathbf{h}^{I} \stackrel{\circ}{=}$ $\left[h_{1}^{I}, h_{2}^{I}, \ldots, h_{K}^{I}\right]$, respectively. The corresponding FIM is given by

$$
\mathbf{J}_{\boldsymbol{\eta}}=\left[\begin{array}{cccc}
\mathbf{J}_{\boldsymbol{\tau}, \boldsymbol{\tau}} & \mathbf{J}_{\boldsymbol{\tau}, \boldsymbol{\theta}} & \mathbf{J}_{\boldsymbol{\tau}, \mathbf{h}^{R}} & \mathbf{J}_{\boldsymbol{\tau}, \mathbf{h}^{I}} \\
\mathbf{J}_{\boldsymbol{\theta}, \boldsymbol{\tau}} & \mathbf{J}_{\boldsymbol{\theta}, \boldsymbol{\theta}} & \mathbf{J}_{\boldsymbol{\theta}, \mathbf{h}^{R}} & \mathbf{J}_{\boldsymbol{\theta}, \mathbf{h}^{I}} \\
\mathbf{J}_{\mathbf{h}^{R}, \boldsymbol{\tau}} & \mathbf{J}_{\mathbf{h}^{R}, \boldsymbol{\theta}} & \mathbf{J}_{\mathbf{h}^{R}, \mathbf{h}^{R}} & \mathbf{J}_{\mathbf{h}^{R}, \mathbf{h}^{I}} \\
\mathbf{J}_{\mathbf{h}^{I}, \boldsymbol{\tau}} & \mathbf{J}_{\mathbf{h}^{I}, \boldsymbol{\theta}} & \mathbf{J}_{\mathbf{h}^{I}, \mathbf{h}^{R}} & \mathbf{J}_{\mathbf{h}^{I}, \mathbf{h}^{I}}
\end{array}\right]
$$

We adopted orthogonal scheme, where each MS is allocated non-overlapping degrees of freedom. Further, AoAs of the different MSs can be considered distinct and the receive beamforming at the BS consist of non-overlapping steering vectors i.e., $\left\|\mathbf{a}_{R}^{H}\left(\theta_{u}\right) \mathbf{a}_{R}\left(\theta_{u}\right)\right\| \gg\left\|\mathbf{a}_{R}^{H}\left(\theta_{u}\right) \mathbf{a}_{R}\left(\theta_{v}\right)\right\| \forall u \neq v$. Hence, the unknown channel parameters of each MS can be estimated independently. To simplify the illustration, but without loss of generality, we can reorder the unknown channel parameters in vector $\boldsymbol{\eta}$ as

$$
\boldsymbol{\eta}=\left[\boldsymbol{\eta}_{1}, \boldsymbol{\eta}_{2}, \ldots, \boldsymbol{\eta}_{K}\right]
$$

where $\boldsymbol{\eta}_{k}$ represents the unknown channel parameters of $k^{t h}$ MS, given by

$$
\boldsymbol{\eta}_{k}=\left[\tau_{k}, \theta_{k}, h_{k}^{R}, h_{k}^{I}\right],
$$

and corresponding FIM for $k^{t h}$ MS can be written as

$$
\mathbf{J}_{\boldsymbol{\eta}_{k}}=\left[\begin{array}{llll}
\mathbf{J}_{\tau_{k}, \tau_{k}} & \mathbf{J}_{\tau_{k}, \theta_{k}} & \mathbf{J}_{\tau_{k}, h_{k}^{R}} & \mathbf{J}_{\tau_{k}, h_{k}^{I}} \\
\mathbf{J}_{\theta_{k}, \tau_{k}} & \mathbf{J}_{\theta_{k}, \theta_{k}} & \mathbf{J}_{\theta_{k}, h_{k}^{R}} & \mathbf{J}_{\theta_{k}, h_{k}^{I}} \\
\mathbf{J}_{h_{k}^{R}, \tau_{k}} & \mathbf{J}_{h_{k}^{R}, \theta_{k}} & \mathbf{J}_{h_{k}^{R}, h_{k}^{R}} & \mathbf{J}_{h_{k}^{R}, h_{k}^{I}} \\
\mathbf{J}_{h_{k}^{I}, \tau_{k}} & \mathbf{J}_{h_{k}^{I}, \theta_{k}} & \mathbf{J}_{h_{k}}^{I}, h_{k}^{R} & \mathbf{J}_{h_{k}^{I}, h_{k}^{I}}
\end{array}\right],
$$

where each entry in (15) is computed as

$$
\mathbf{J}_{u, v}=\frac{1}{\sigma^{2}} \int_{0}^{T_{s}} \Re\left\{\frac{\partial u^{*}(t)}{\partial u} \frac{\partial u(t)}{\partial v}\right\} d t,
$$

and $u(t)$ is the deterministic part of the received signal. For the $k^{t h} \mathrm{MS}, u(t)$ is given by

$$
u(t) \stackrel{\circ}{=} \mathbf{w}^{H} \mathbf{h}_{k} x_{k}\left(t-\tau_{k}\right) .
$$

Following [5] and [8], the entries are calculated as

$$
\begin{aligned}
\mathbf{J}_{\tau_{k}, \tau_{k}} & =\frac{P_{c}}{\sigma^{2}}\left|h_{k}\right|^{2}\left(2 \pi \Delta_{f}\right)^{2} n^{2}\left|q_{k}\right|^{2}, \\
\mathbf{J}_{\theta_{k}, \theta_{k}} & =\frac{P_{c}}{\sigma^{2}}\left|h_{k}\right|^{2}\left|\dot{q}_{k}\right|^{2}, \\
\mathbf{J}_{\theta_{k}, \theta_{k}} & =\frac{P_{c}}{\sigma^{2}} \Re\left\{h_{k}^{*} q_{k} \dot{q}_{k}^{*}\right\}, \\
\mathbf{J}_{\theta_{k}, \theta_{k}} & =\frac{P_{c}}{\sigma^{2}} \Re\left\{j h_{k}^{*} q_{k} \dot{q}_{k}^{*}\right\}, \\
\mathbf{J}_{h_{k}^{R}, h_{k}^{R}} & =\frac{P_{c}}{\sigma^{2}}\left|q_{k}\right|^{2}, \\
\mathbf{J}_{h_{k}^{I}, h_{k}^{I}} & =\frac{P_{c}}{\sigma^{2}}\left|q_{k}\right|^{2},
\end{aligned}
$$

where $*$ indicates the complex conjugate, $q_{k}=\mathbf{w}^{H} \mathbf{a}_{R}\left(\theta_{k}\right)$ and $\dot{q}_{k}=\mathbf{a}_{R}^{H}\left(\theta_{k}\right) \mathbf{D}_{k}^{H} \mathbf{w}$, in which $\mathbf{D} \in \mathbb{C}^{N_{B} \times N_{B}}$ is a diagonal matrix with the $l^{t h}$ entry given by $D^{l}=j \pi(l-1) \cos \left(\theta_{k}\right) . P_{c}$ is the power per sub-carrier and $\Delta_{f}$ is inter carrier spacing for OFDM frame. In addition, all the remaining entries are zero.

\section{B. CRB for Position}

It is shown in [5] and [8], CRB for the position of $k^{t h}$ MS can be calculated from the inverse of FIM $\mathbf{J}_{\tilde{\boldsymbol{\eta}}_{k}}$ associated with the $\tilde{\boldsymbol{\eta}}_{k} \stackrel{\circ}{=}\left[p_{x}^{k}, p_{y}^{k}, h_{k}^{R}, h_{k}^{I}\right]$. We first perform parameter transformation from $\boldsymbol{\eta}_{k}$ to $\tilde{\boldsymbol{\eta}}_{k}$ using the geometric relationship, as shown in Figure 1. For the $k^{t h} \mathrm{MS}, \boldsymbol{\eta}_{k}$ and $\tilde{\boldsymbol{\eta}}_{k}$ are related by: $\tau_{k}=\left\|\mathbf{q}-\mathbf{p}_{k}\right\| / c, \cos \left(\pi-\theta_{k}\right)=\left(p_{x}^{k}-q_{x}^{B S}\right) /\left\|\mathbf{q}-\mathbf{p}_{k}\right\|$ and $\sin \left(\pi-\theta_{k}\right)=\left(p_{y}^{k}-q_{y}^{B S}\right) /\left\|\mathbf{q}-\mathbf{p}_{k}\right\|$. Therefore, by means of bijective transformation $\mathbf{T} \in \mathbb{R}^{4}, \mathbf{J}_{\tilde{\boldsymbol{\eta}}_{k}}$ can be calculated as

$$
\mathbf{J}_{\tilde{\boldsymbol{\eta}}_{k}}=\mathbf{T} \mathbf{J}_{\boldsymbol{\eta}_{k}} \mathbf{T}^{H}
$$

where

$$
\mathbf{T} \doteq \frac{\partial \boldsymbol{\eta}_{k}^{T}}{\partial \tilde{\boldsymbol{\eta}}_{k}}
$$

in which

$$
\begin{aligned}
\frac{\partial \tau_{k}}{\partial \mathbf{p}_{k}} & =\frac{1}{c}\left[\cos \left(\pi-\theta_{k}\right), \sin \left(\pi-\theta_{k}\right)\right]^{T}, \\
\frac{\partial \theta_{k}}{\partial \mathbf{p}_{k}} & =\frac{1}{\left\|\mathbf{q}-\mathbf{p}_{k}\right\|}\left[-\sin \left(\pi-\theta_{k}\right), \cos \left(\pi-\theta_{k}\right)\right]^{T},
\end{aligned}
$$

and $\partial h_{k}^{R} / \partial h_{k}^{R}=\partial h_{k}^{I} / \partial h_{k}^{I}=1$. All the remaining terms in $\mathbf{T}$ are zero.

\section{Simulation Results}

This section presents numerical results to demonstrate tradeoff between effective data-rate and position bound. In particular, we analyze the impact of increasing training overhead on positioning accuracy and achievable data-rate as well as quantify the trade-off between these two metrics. 


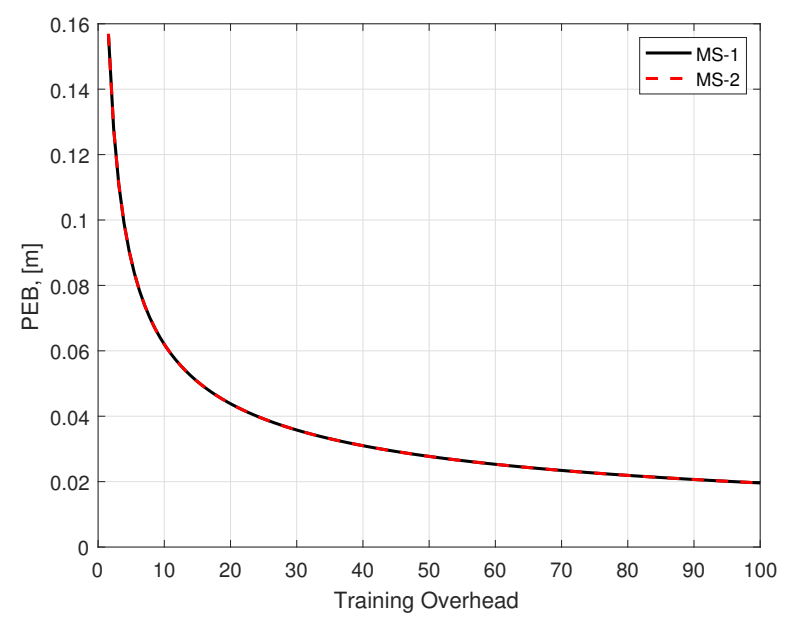

Fig. 3. Performance of the PEB as a function of beam training overhead with exhaustive beam training strategy.

\section{A. Simulation setup}

We consider an idealized MIMO orthogonal-frequencydivision-multiplexing (OFDM) communication system with 1024 sub-carriers, inter-carrier spacing $\Delta_{f}=75 \mathrm{kHz}$, timefrequency efficiency is equal to one and the carrier-frequency is set to $28 \mathrm{GHz}$. Without loss of generality, we adopted DFT codebook based RF beam training at the BS. Let $\mathcal{W}$ be the RF beamforming codebook with cardinality $|\mathcal{W}|=N_{B}$. We consider RF beamforming only in the azimuth plan, although similar analysis could be extended to elevation direction as well. Then, one beam during beam training corresponds to one OFDM symbol and we assume $T_{f}=256$ OFDM symbols.

Unless stated otherwise, we consider two MSs located at a fixed distance of 30 meters to the BS with each MS having its own channel properties. We study the case, $\theta_{1}=0^{\circ}$ and $\theta_{2}=50^{\circ}$ with a fixed transmission power $P_{t x}=27 \mathrm{dBm}$. Further, identical requirements for data-rate $R_{o}$ and position accuracy $Q_{t h}$ are applied to both MSs.

\section{B. Trade-off between PEB and training overhead}

The first result, illustrated in Figure 3, shows the position error bound (PEB) as a function of increasing training overhead with the exhaustive beam training strategy. Positioning accuracy improves with the increase in the number of sounded beams, as more Fisher information lead to lower PEB. Clearly, longer the training, lower the positioning error. Furthermore, because of orthogonal reception and with the sufficiently large density of receive beamforming vectors at the BS, PEB performance is independent of individual AoA.

\section{Trade-off between sum-rate and training overhead}

Figure 4 shows the achievable sum-rate as a function of training overhead with the constraint of identical data-rate requirement for both MSs. In contrast to PEB show in Figure 3, we note that, there exist an optimum receive beamforming

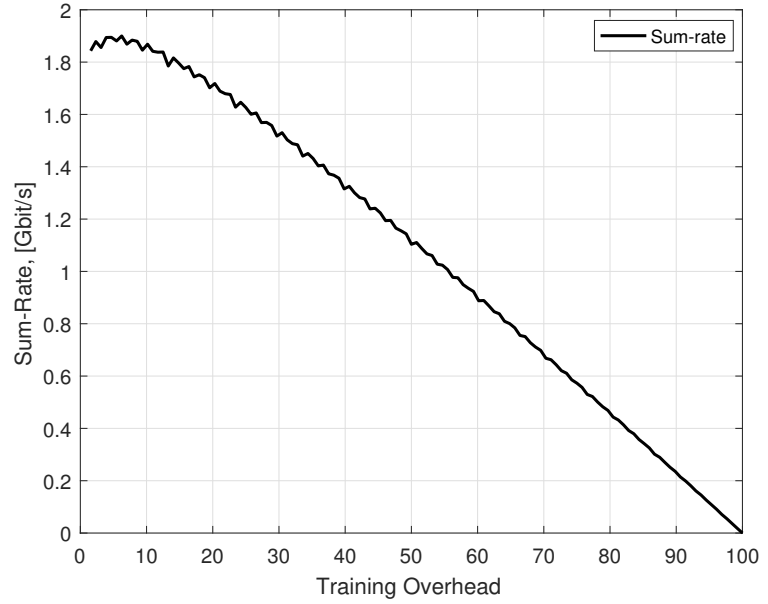

Fig. 4. Achievable sum-rate as a function of training overhead with identical data-rate requirements for each MS.

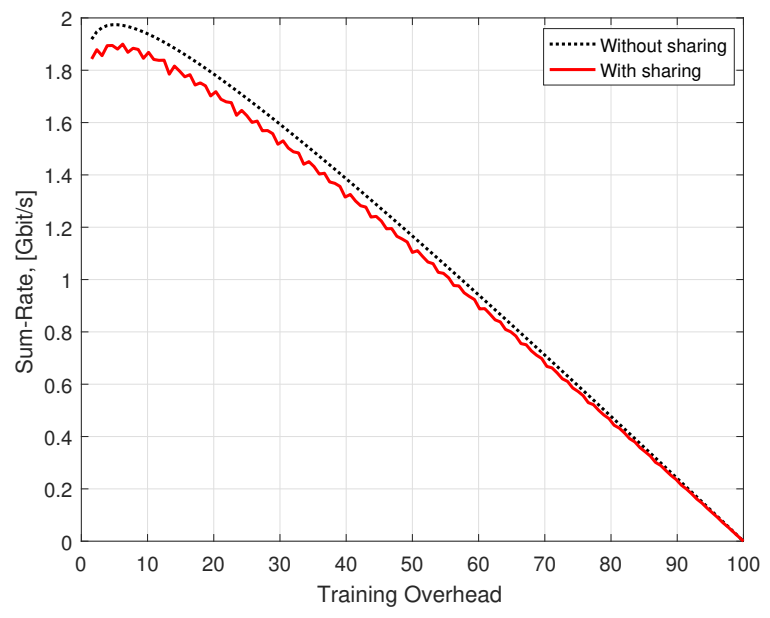

Fig. 5. Comparison between achievable sum-rate under different allocation schemes; solid curve: sharing of $T_{d}$ with compromise receive beam, dotted curve: selecting best MS and allocation all $T_{d}$ with the optimal receive beam.

codebook size that achieves maximum sum-rate. For the considered simulation assumptions, sum-rate is maximized when the training overhead is $6.25 \%$, that corresponds to $N_{B}=16$.

As mentioned in Section II, for the uplink data reception, we use a common receive beam $\tilde{\mathbf{w}}$ that is obtained by the superposition of best receive beams of each MS and then we find the optimal sharing strategy on $T_{d}$ such that both MS receive equivalent data-rates. Figure 5 illustrate the loss in sum-rate because of the compromise receive beam $\tilde{\mathbf{w}}$ and sharing of $T_{d}$ among MSs. The dotted curve in Figure 5 is obtained by aligning the receive beamforming only in the direction of the best MS and allocating all the degrees of freedom to the selected MS. 


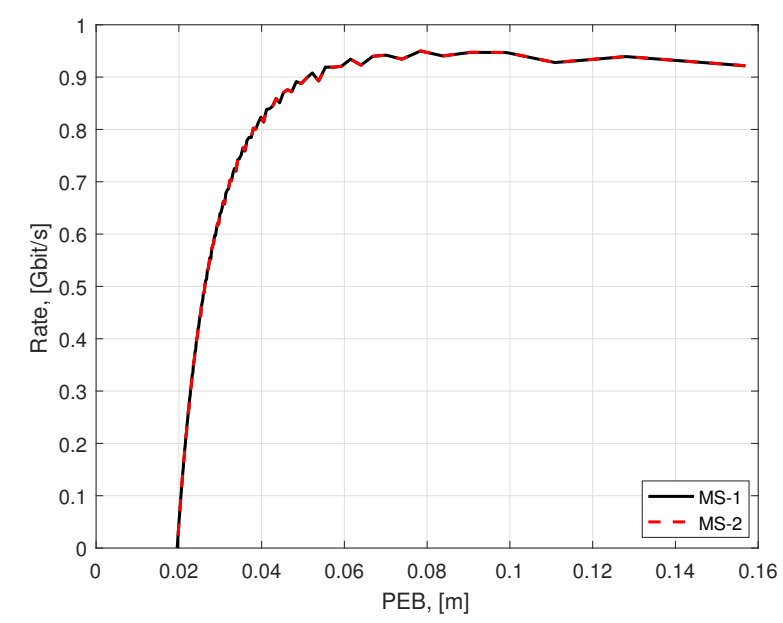

Fig. 6. Trade-off between PEB and the effective data-rate when varying the training overhead.

\section{Positioning and sum-rate trade-off}

Figure 6 shows the relationship between effective data-rate and positioning accuracy of both MSs. The curves overlap because of our assumption on identical data-rate requirements and the performance of PEB is invariant to $\theta$. The small fluctuation in the curve represent the beam misalignment due to relative location of BS and MSs, which is more noticeable with the smaller codebook. It can be noted, that the maximum datarate is achieved with the approximate positioning accuracy of $7.85 \mathrm{~cm}$. This is comparable with the state-of-art GPS based localization and other similar methods, which require signals from several stations.

\section{E. PEB performance with increasing number of $M S s$}

Finally, we illustrate the performance PEB with increasing number of MS. For the result shown in Figure 7, we consider a fixed beamforming codebook of size $N_{B}=8$, a fixed duration for training phase $T_{t}=0.5 T_{f}$ which corresponds to 128 OFDM symbols, and further training symbols are equally divided among all active MSs. We assume, all MSs are randomly located with a fixed distance of 30 meters to the BS. Since the positioning accuracy improves with an increase in the collected Fisher information, that is related to the training symbols allocated to each MS. Clearly, with the increase in number of MS, the effective training duration for each MS decreases and hence results in lower Fisher information and that leads to the higher values of PEB, as shown in Figure 7 for the $k^{t h}$ MS.

\section{CONCLUSiON}

In this paper, we study and quantify the trade-off between positioning accuracy and achievable data-rate for a mmWave communication in uplink multi-user scenario. Simulation results manifest the conflicting implication of two performance metrics: PEB improves with the increase in number of training

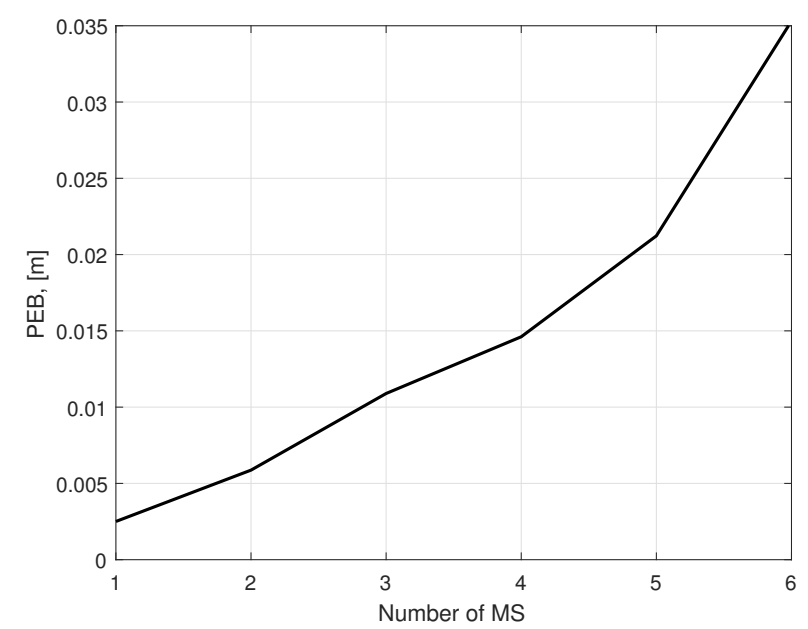

Fig. 7. Performance of the PEB as a function of increasing number of MS assuming a fixed training overhead and receive beamforming codebook.

beams but results in lower data-rate. It can be concluded, maximum data-rate is achieved with a centimetre level positioning accuracy. For the future work, interest is to include random sources of beam misalignments such as blockers and extension to multi-cell multi-user scenario.

\section{ACKNOWLEDGMENTS}

The research leading to these results has received funding from the Academy of Finland projects: Fundamental of Simultaneous Localization and Communications (FALCON) and Positioning-aided Reliably-connected Industrial Systems with Mobile mmWave Access (PRISMA).

\section{REFERENCES}

[1] M. Mueck, et al., "5G CHAMPION - Rolling out 5G in 2018," in Proc. IEEE Global Commun. Conf. Workshops, Dec. 2016, pp. 1-6.

[2] T. S. Rappaport, et.al., "Millimeter Wave Mobile Communications for 5G Cellular: It Will Work!" IEEE Access, vol. 1, pp. 335-349, 2013.

[3] J. G. Andrews, et.al., "What Will 5G Be?" IEEE J. Sel. Areas Commun., vol. 32, no. 6, pp. 1065-1082, Jun. 2014.

[4] A. Dammann, R. Raulefs, and S. Zhang, "On Prospects of Positioning in 5G," in Proc. IEEE Int. Conf. Commun. Workshop, 2015, pp. 12071213.

[5] A. Shahmansoori, G. E. Garcia, G. Destino, G. Seco-Granados, and H. Wymeersch, "5G Position and Orientation Estimation through Millimeter Wave MIMO," in Proc. IEEE Global Commun. Conf. Workshops, Dec. 2015, pp. 1-6.

[6] S. Jeong, O. Simeone, A. Haimovich, and J. Kang, "Beamforming Design for Joint Localization and Data Transmission in Distributed Antenna System," IEEE Trans. Veh. Technol., vol. 64, no. 1, pp. 6276, Jan. 2015.

[7] J. C. Aviles and A. Kouki, "Position-Aided mm-Wave Beam Training Under NLOS Conditions," IEEE Access, vol. 4, pp. 8703-8714, 2016.

[8] G. Destino and H. Wymeersch, "On the Trade-off Between Positioning and Data-Rate for mm-Wave Communications," in Proc. IEEE Int. Conf. Commun. Workshop, May 2017.

[9] J. Saloranta, G. Destino, and H. Wymeersch, "Comparison of different beamtraining strategies from a rate-positioning trade-off perspective," in Proc. Eur. Conf. Networks and Commin., Jun. 2017, pp. 1-5.

[10] J. Saloranta and G. Destino, "Reconfiguration of 5G radio interface for positioning and communication," in Proc. European Sign. Process. Conf., Aug. 2017, pp. 898-902.

[11] S. M. Kay, "Fundamentals of statistical signal processing. Vol 1, Estimation theory," 1993. 